\title{
Control glucémico, autocuidado y estrés en pacientes con diabetes mellitus tipo 2 residentes de Monterrey, México.
}

\author{
Abraham Arias-González ${ }^{1, a}$, Milton Carlos Guevara Valtier ${ }^{1, b}$, María de los Ángeles Paz-Morales ${ }^{1, b}$, San- \\ draValenzuela-Suazuo ${ }^{2, c}$, Valentina Rivas-Acuña ${ }^{3, d}$
}

\section{RESUMEN}

La diabetes mellitus es una enfermedad crónica de alta prevalencia en México en quienes la padecen el autocuidado de la enfermedad y el manejo del estrés influyen en el auto control de dicha patología.Objetivos: Conocer el control glucémico, autocuidado y estrés en pacientes con diabetes mellitus tipo 2 en una muestra de pacientes que acuden a un centro especializado en Diabetes Mellitus ubicado en la ciudad de Monterrey, México. Material y Métodos: Se utilizó un diseño descriptivo, la muestra se conformó por un total de 122 pacientes que acudieron a un centro especializado en el cuidado de la diabetes. Se recurrió a la medición de cintura e índice de masa corporal, se aplicaron cuestionarios de lápiz y midió la glucosa en sangre periférica. Resultados: Se identificaron valores medios de cintura en hombres y mujeres de 96,45 y $88,65(\mathrm{DE}=10,83$ y 17,01 ; respectivamente), índice de masa corporal de 29,57 $(20,42-53,75)$, estrés percibido 42,76 $(12,34)$, estrés en diabetes $44,31(24,74)$ y en cuanto a las acciones de autocuidado en diabetes la media identificada fue de $42,01(18,64)$. Conclusiones: La mayoría de los participantes demostraron un buen control de la enfermedad, aunque su nivel de autocuidado es bajo. En cuanto al estrés percibido y en diabetes los niveles fueron bajo y moderado respectivamente.

PALABRAS CLAVE: Diabetes mellitus, autocuidado, estrés.

\section{Glycemic control, self-care and stress in patients with type 2 diabetes mellitus residents of Monterrey, Mexico.}

\section{SUMMARY influence self-control}

Diabetes mellitus is a chronic disease with high prevalence in Mexico in those with self-management of the disease and stress management influence self-control of this pathology. Objectives: To know the glycemic control, self-care and stress in patients with diabetes mellitus type 2 who presented in a specialized center in Diabetes Mellitus located in the Monterrey city of Mexico. Material and Methods: A descriptive design was used; the sample was shaped for 122 patients who were attended in a specialized center in diabetes care. It was resorted for measuring wrist and BMI, the pencil questionnaires were applied and the glucose was measured in peripheral blood. Results: The average values of wrist in women and men were identified of 96.45 y 88.65 ( $\mathrm{DE}=10.83$ y 17.01; respectively), the BMI of 29.57 (20.42 - 53.75), perceived stress 42.76 (12.34), the stress in diabetes 44.31 (24.74) and according to the actions of self-care in diabetes the identified average was 42.01 (18.64). Conclusions: The most of the participants showed a good disease control, although their self-care levels were low. According the perceived stress and the diabetes, the levels were low and moderate respectively.

KEY WORDS: Diabetes mellitus, self-care, stress.

${ }^{1}$ Facultad de Enfermería, Universidad Autónoma de Nuevo León. Nuevo León, México.

${ }^{2}$ Universidad de Concepción. Concepción, Región del Bío Bío, Chile.

${ }^{3}$ Universidad Juarez Autónoma de Tabasco. Villahermosa, Tabasco, México.

${ }^{a}$ Maestro en Ciencias de Enfermería ; ${ }^{b}$ Doctor en Ciencias de la Educación ; ${ }^{\mathrm{c}}$ Doctora en enfermería ; ${ }^{\mathrm{D}}$ Doctora en Ciencias de Enfermería. 


\section{INTRODUCCIÓN}

La Diabetes Mellitus Tipo 2 (DT2) es una enfermedad crónico-degenerativa, caracterizada por hiperglucemia resultante de un defecto en la secreción o acción de la insulina o bien de ambas. El padecer DT2 a temprana edad, expone a la persona a períodos más largos que aumentan la probabilidad de que se presenten altos niveles de glucosa en sangre incrementando el riesgo y posibilidad de presentar complicaciones micro y macrovasculares relacionadas con un deficiente control glucémico (1-6).

La prevalencia de DT2 en México ha tenido un incremento significativo en los últimos años; en el 2006 la prevalencia de adultos ya conocidos con esta afección fue de 7,0\% y para el año 2012 tuvo un aumento del 1,8\%, resultando en una prevalencia de 9,2\%. Estas cifras sitúan la afección como una de las principales causas de morbilidad, discapacidad prematura y mortalidad en población Mexicana (7).

La DT2 se diagnostica en la mayoría de los casos después de los 30 años de edad, coexistiendo con la presencia de obesidad que a su vez es el factor de riesgo más alto para desarrollar DT2, además de los antecedentes familiares y una vida sedentaria.De acuerdo a la Encuesta Nacional de Salud Alimentaria del total de consultas proporcionadas por los servicios públicos de salud ambulatorios, $61,1 \%$ tuvo como motivo la DT2. Esto sugiere altos costos para los servicios de salud, ya que aplican de dos y tres veces más recursos a personas con enfermedades crónico-degenerativas entre las que figura la DT2. Además se reportó que solo el $25 \%$ de la población tuvo cifras de HbA1c correspondientes a "control glucémico"( 1,2$)$.

El control glucémico según la ADA son las cifras de glucosa en sangre en ayuno entre 70 y $130 \mathrm{mg} / \mathrm{dL}$, o una hemoglobina glucosilada $(\mathrm{Hb} 1 \mathrm{Ac}) \leq 7$ lo que es posible lograr mediante acciones de autocuidado como una alimentación saludable, actividad física y ejercicio suficiente para mantener un equilibrio entre consumo y gasto calórico, tomar los medicamentos ordenados y auto-monitoreo de la glucosa (1). Para lograr los rangos mencionados anteriormente se requieren cambios en los estilos de vida de las personas con DT2 lo cual es complicado ya que implica modificar hábitos de toda una vida, produciendo a menudo una carga importante de estrés. Diversos estudios muestran que el estrés se encuentra relacionado negativamente con las actividades para manejar el tratamiento en personas con DT2 por el impacto biológico y emocional asociados a la enfermedad (8-12).

Por otro lado, el estrés relacionado con la diabetes se ha estudiado para conocer la relación que tiene con el control glucémico, en este sentido se ha identificado una relación positiva entre el estrés relacionado con la enfermedad y el control glucémico, lo que significa que a mayor estrés, cifras más elevadas de $\mathrm{HbAlc}$, o bien se produce un descontrol por minimizar las actividades de AC llegando a afectar negativamente la calidad de vida de los enfermos. Se conoce que en el sujeto con DT2 al percibir situaciones estresantes ocurre una interacción de hormonas como el cortisol, la adrenalina y el glucagón por lo que éstas ejercen un aumento de glucosa en sangre aún más de lo que pudieran manifestarse $(13,14)$.

La importancia de estudiar el estrés en las personas con DT2 radica en que éste es inducido por situaciones emocionales como la falta de conocimiento sobre la enfermedad, miedo, angustia y de tipo biológicas como la interacción de distintas hormonas que alteran las necesidades de insulina del sujeto y elevan las cifras de glucosa en sangre. El estrés psicológico puede generar una respuesta de huida en los individuos llevándolos a consumir una mayor cantidad de carbohidratos, a que no duerman o manifiesten desapego en el consumo de medicamentos indicados, favoreciendo el descontrol glucémico (1).

El objetivo del presente estudio fue conocer el control glucemico, autocuidado y estrés en pacientes con diabetes mellitus tipo 2 en una muestra de derechohabientes que acuden a un centro especializado en Diabetes Mellitus ubicado en la ciudad de Monterrey, México.

\section{MATERIAL Y MÉTODOS}

El diseño de la investigación fue de tipo descriptivo, la población estuvo conformada por personas adultas de ambos sexos con diagnóstico de DT2, que asistían a la consulta de control en una Institución especializada en Diabetes ubicada en la ciudad de Monterrey, N.L. México. Se recurrió a la utilización de un muestreo por conveniencia (15). El tamaño de la muestra se estimó por el paquete estadístico nQueryAdvisor V4.0 ${ }^{\circledR}$, con un nivel de potencia de $90 \%$, un nivel de significancia de 0,05 y una $\mathrm{R}^{2}=0,09$. El tamaño de la muestra resultante fue de 122 adultos con DT2 entre 18 y 60 años de edad y que pudieran ser capaces de leer y escribir.Se excluyeron mujeres con diabetes gestacional, con diagnostico de Diabetes Tipo 1.

Para evaluar la variable estrés las mediciones se realizaron con dos escalas, la primera evaluaestrés percibido EEP-14; y la segunda evaluael estrés producto de la diabetes.Se aplico un cuestionario de acciones de cuidado en diabetes para medir las actividades de auto-cuidado que realiza la persona con DT2 (dieta, ejercicio, medicamentos y monitoreo de la glucosa) (16-19). 
Aunado a las escalas utilizadas se documentaron variables sociodemograficas y clínicas en una cedula especial, las mediciones antropométricas como el peso y talla fueron auxiliadas con el uso de una báscula clínica IRONMAN ${ }^{\circledR-}$ modelo BC554 y se calculo el índice de masa corporal con base a los criterios de la Organización Mundial de la Salud (Tabla 1). Con el fin de valorar el control glucemico se realizaron pruebas glucosa sanguínea periférica a personas que cumplieran con un mínimo de 8 horas de ayuno, se considero control glucémico adecuado a resultados entre 70 y $130 \mathrm{mgs} / \mathrm{dL}(20)$.

Previo a cualquier procedimiento se contó con la aprobación de la institución auspiciadora y de la especializada en diabetes, se obtuvieron los listados generales de pacientes con DT2 de los que se diseño una segunda lista con quienes cumplían con los criterios de inclusión, mismos que se contactaron vía telefónica o durante su cita de consulta a la institución especializada en diabetes. El procesamiento de los datos estadísticos se realizó electrónicamente en el paquete estadístico SPSS versión 21 para Mac ${ }^{\circledR}$ y se utilizaron medidas de tendencia central, frecuencias y porcentajes.

\section{RESULTADOS}

Los datos descriptivos de las variables demográficas del estudio fueron los siguientes: el promedio de edad fue de 48,15 años $(D E=11,17)$ y $10,59(D E=4,08)$ años de escolaridad, El 69\% fueron mujeres y la mayoría se dedicaban al hogar o eran desempleados $(54,2 \%)$, así mismo vivían en Monterrey, México (56\%).

En cuanto a los datos que dan respuesta a los objetivos de investigación se encontró una media de 29,57 $(D E=5,24)$ en el índice de masa corporal este indicador se clasificó según puntos de corte establecidos por la Organización Mundial de la Salud encontrando que sólo el 14,8\% presentaron cifras normales (IMC $<25)$; el resto $(85,2 \%)$ se clasificaron con sobrepeso $(43,4 \%) \mathrm{u}$ obesidad $(41,8 \%)$. Cabe aclarar que las mujeres tuvieron el porcentaje más alto de obesidad $(69,8 \%)$ comparadas con los hombres.

Tabla 1. Clasificación del IMC según la OMS.

\begin{tabular}{cc}
\hline & Clasificación \\
\hline Peso normal & $\mathrm{IMC} \leq 25$ \\
Sobrepeso & $\mathrm{IMC} \geq \mathrm{a} 25,1 \mathrm{~kg} / \mathrm{m}^{2} \mathrm{y} \leq \mathrm{a} 29,9 \mathrm{~kg} / \mathrm{m}^{2}$ \\
Obesidad & $\mathrm{IMC} \geq \mathrm{a} 30 \mathrm{~kg} / \mathrm{m}^{2}$ \\
\hline
\end{tabular}

Fuente: Cedula de datos sociodemográficos, antropométricos y bioquímicos.

$\mathrm{n}=122$
Tabla 2. Características antropométricas y bioquímicas de los participantes.

\begin{tabular}{|c|c|c|c|c|}
\hline Variables & X (DE) & Mediana & $\begin{array}{c}\text { Valor } \\
\text { Mínimo }\end{array}$ & $\begin{array}{c}\text { Valor } \\
\text { Máximo }\end{array}$ \\
\hline Peso kg & $\begin{array}{c}78,64 \\
(15,46)\end{array}$ & 78,00 & 51,90 & 137,60 \\
\hline Talla cm & $\begin{array}{c}162,97 \\
(8,34)\end{array}$ & 162,00 & 138,00 & 186,00 \\
\hline Cintura & $\begin{array}{c}90,92 \\
(16,14)\end{array}$ & 90,00 & 58,00 & 148,00 \\
\hline Hombres & $\begin{array}{c}96,45 \\
(10,83)\end{array}$ & 94,50 & 79,00 & 118,00 \\
\hline Mujeres & $\begin{array}{c}88,65 \\
(17,01)\end{array}$ & 88,00 & 42,00 & 148,00 \\
\hline IMC & $\begin{array}{l}29,57 \\
(5,24)\end{array}$ & 28,58 & 20,42 & 53,75 \\
\hline Glucosa & $\begin{array}{l}126,09 \\
(53,60)\end{array}$ & 106,50 & 67,00 & 346,00 \\
\hline
\end{tabular}

Fuente: Cedula de datos sociodemográficos, antropométricos y bioquímicos.

$\mathrm{n}=122$

La obesidad abdominal indicada por la circunferencia de cintura tuvo un comportamiento semejante, encontrando que sólo el 27,9\% presentaron cifras de normalidad (hombres $>90 \mathrm{~cm} /$ mujeres $>80 \mathrm{~cm})$; el resto $(72,1 \%)$ presento obesidad abdominal; igualmente las mujeres presentaron el porcentaje más alto. Con respecto a la glucosa sanguínea se encontró una media de $126,09 \mathrm{mgs} / \mathrm{dL}(D E=53,60)$ es decir el $64,8 \%$ de los participantes tuvo cifras de glucosa plasmática en ayuno consideradas como buen control (70-130 mgs/dL) (Tabla 2) (21).

Las escalas estrés percibido y en diabetes; denotaron puntajes que ubicaban a la muestra en niveles bajo y moderado, respectivamente. El auto-cuidado ejercido por los adultos con DM2, se consideró bajo; al analizar las sub-escalas se observa que el ejercicio y el auto-monitoreo se reportan con promedios aún más bajos que la media obtenida de la escala general. El puntaje medio de la escala SF-12 sugiere que en general la muestra se auto percibe con un buen estado de salud (Tabla 3 ).

\section{DISCUSIÓN}

Una de las características de la muestra estudiada es que un alto porcentaje de los participantes reportaron cifras de glucosa plasmática en control glucémico $(64,8 \%)$ con glucemias entre $70-130 \mathrm{mg} / \mathrm{dL}$. Posiblemente este resultado pudiera deberse a que los participantes tienen conocimiento sobre cómo llevar a cabo su tratamiento; el promedio de educación formal que reporto la población estudiada fue de 10 años lo que significa un nivel de edu- 
TABLA 3. Datos descriptivos de las escalas utilizadas.

\begin{tabular}{lccc}
\hline \multicolumn{1}{c}{ Escalas } & $\boldsymbol{X}(\boldsymbol{D E})$ & $\begin{array}{c}\text { Valor } \\
\text { Mínimo }\end{array}$ & $\begin{array}{c}\text { Valor } \\
\text { Máximo }\end{array}$ \\
\hline Estrés percibido & $\begin{array}{c}42,76 \\
(12,34)\end{array}$ & 8,93 & 71,43 \\
Estrés en diabetes & $\begin{array}{c}44,31 \\
(24,74)\end{array}$ & 0,00 & 96,47 \\
Acciones de & 42,01 & & \\
autocuidado en & $(18,64)$ & 0,00 & 88,57 \\
diabetes & 52,75 & 0,00 & 100,00 \\
\multicolumn{1}{c}{ Dieta } & $(21,95)$ & & \\
\multicolumn{1}{c}{ Ejercicio } & 28,57 & 0,00 & 100,00 \\
& $(30,44)$ & & \\
\multicolumn{1}{c}{ Auto-monitoreo } & 28,80 & 0,00 & 100,00 \\
& $(29,51)$ & 0,00 & \\
\multicolumn{1}{c}{ Medicamentos } & 35,64 & 4,76 & 66,67 \\
& $(14,54)$ & & \\
\hline
\end{tabular}

Fuente: Escalas de medición $\mathrm{n}=122$

cación media superior; además la mayoría de la muestra cuenta con seguridad social por contar con un empleo, esta situación podría sugerir que algunos participantes reciben sistemáticamente el tratamiento para la DT2, facilitando un control de la enfermedad.

Orem describe que el AC es una acción intencionada y dirigida que es sensible a los conocimientos de las personas sobre cómo llevar a cabo el cuidado y desarrollo de la vida humana, la salud y el bienestar en las condiciones y circunstancias diferentes; pudiéndose explicar de esta forma un beneficio que tendrían los participantes por poseer un buen nivel de escolaridad (22).

En la mayoría de los participantes se encontró que padecen de obesidad general y abdominal; resaltando que las mujeres presentan mayor índices de obesidad tanto por IMC como por abdominal; estas cifras concuerdan con lo reportado en la ENSA y Barquera, Campos, Hernández, Pedroza y Rivera (23) donde reportaron que el 71,3\% tiene obesidad general y el $74 \%$ sufría obesidad abdominal, siendo mayor en mujeres que en los hombres.

Se identificó bajo nivel de estrés percibido en la muestra estudiada; pudiéndose explicar en parte al nivel de escolaridad que tienen los participantes como un factor protector, concordando con el estudio de Toledano et al., (24), sin embargo el estrés relacionado con la diabetes específicamente el asociado al médico se encontró alto, esto podría deberse al tipo de relación que tiene el paciente con su personal de salud o bien la información que el médico pudiera no aclarar al paciente sobre su tratamiento, los resultados concuerdan con lo reportado por Durán, Rivera y Franco (25), la falta de información por parte del médico hacia el paciente aumentan el estrés relacionado con la diabetes y la cifra de $\mathrm{HbA} 1 \mathrm{c}$ fuera de parámetros normales.

Se identificó un bajo nivel de acciones de auto-cuidado en DT2, Orem (26) en la teoría del AC menciona que este es adquirido como una función reguladora humana ejecutada deliberadamente con cierto grado de complejidad y efectividad, esto podría explicarse a que a mayor complejidad de las acciones de cuidado menor será el apego a dichas acciones que debe realizar el sujeto con DT2. Llama la atención de que a pesar de los bajos puntajes en acciones de $\mathrm{AC}$, la proporción de personas en control glucémico fue alta si se compara contra el $25 \%$ de cifra nacional.

\section{CONCLUSIONES}

Se concluye que la mayoría de los participantes demostraron un buen control de la enfermedad, aunque su nivel de autocuidado es bajo. En cuanto al estrés percibido y en diabetes los niveles fueron bajo y moderado respectivamente.

\section{Correspondencia:}

Milton Carlos Guevara Valtier

Facultad de Enfermería, Universidad Autónoma de Nuevo León

Av. José Eleuterio González (Gonzalitos 1500),

Mitras Centro, Monterrey, N.L.Mexico

Correo electrónico: carlos_valtier7@hotmail.com.mx

\section{REFERENCIAS BIBLIOGRÁFICAS}

1. American Diabetes Association. Standards of medical care in diabetes. Diabetes Care. 2013; 36 (1): 67-74.

2. Secretaría de Salud, Instituto Nacional de Salud Pública. En cuesta Nacional de Salud y Nutrición 2012. Ciudad de Mexico: Instituto Nacional de Salud Pública; 2012 (Citado el 20 enero 2013). Disponible en: http://www.oportunidades. gob.mx:8010/es/wersd53465sdg 1/docs/2012/ ensanut_2012_informe_resultados.pdf

3. Colagiuri R, Brown J, Dain K. Plan mundial contra la diabetes.Brussels, Belgium:Federación Internacional de Diabetes; 2011. p. 27.

4. McGill M, McGuire H. El marco de la fid para la educación diabética: situación actual y perspectivas futuras. Diabetes Voice. 2009 (Citado el 20 enero 2013); 54 (1): 20-

23. Disponible en: http://www.idf.org/sites/default/files/ attachments/2009_1_McGill_McGuire_ES.pdf

5. Mancha C, García A, López D. Datos epidemiológicos actuales de enfermedades crónicas no transmisibles y del 
síndrome metabólico. En: González A. Síndrome metabólico y enfermedad cardiovascular. México DF: Intersistemas; 2012. p. 3-21.

6. Rosas J, LyraR,Cavalcanti N. Diabetes Mellitus: Visión Latinoamericana. México: Intersistemas; 2009. p. 752.

7. Secretaría de Salud, Instituto Nacional de Salud Pública. Encuesta Nacional de Salud y Nutrición 2006 resultados por entidad federativa. Nuevo León: Instituto Nacional de Salud Pública; 2006 (Citado el 6 Febrero del 2013). Disponible en: http://www.insp.mx/images/stories/ Produccion/pdf/ENSANUTEF/nl.pdf

8. Hernández J, Licea M, Hernández P, Marcel E, Quesada M. Estrés Oxidativo y diabetes mellitus. Rev Mex Patol Clin. 2011 (Citado el 6 Febrero 2013); 58 (1): 4-15. Disponible en: http://www.medigraphic.com/pdfs/patol/ pt-2011/pt111b.pdf

9. Montes R, Oropeza R, Pedroza F, Verdugo J, Enríquez J. Manejo del estrés para el control metabólico de personas con diabetes mellitus tipo 2. En-claves del pensamiento. 2013 (Citado el 6 Febrero 2013); 7(13): 67-87. Disponible en: http://www.redalyc.org/articulo.oa?id=141128006004

10. Gomes-Villas BLC, Foss MC, Foss de Freitas MC, Pace AE. Relationship among social support, treatment adherence and metabolic control of diabetes mellitus patients. Rev. LatinoAm. Enfermagem.2012 (Citado el 5 Marzo del 2013); 20(1): 52-58. Disponible en: http://www.scielo.br/scielo.php? script $=$ sci_pid=S010411692012000100008\&lng=en\&nrm $=$ iso\&tlng=en

11. Garay-Sevilla ME, Porras JS, Malacara JM. Coping strategies and adherence to treatment in patients with type 2 diabetes.Revista de investigación clínica. 2011(Citado el 2 de Abril del 2013); 63(2):155-161. Disponible en: http:// www.medigraphic.com/pdfs/revinvcli/nn-2011/nn112g.pdf

12. Quirós-Morales D, Villalobos-Pérez A. Comparación de factores vinculados a la adherencia al tratamiento en diabetes mellitus tipo II entre una muestra urbana y otra rural de Costa Rica. Revista de Psicología de Colombia. 2007 (Citado el 8 Abril del 2013); 6(3):679-688. Disponible en: http:/www.scielo.org.co/pdf/rups/v6n3/v6n3a17.pdf

13. Egede L, Dismuke C. Serious psychological distress and diabetes: a review of the literature. Curr Psychiatry Rep. (2012); 14 (1): 15-22.

14. Surwit R, Van Tilburg M, Zucker N, et al. Stress management improvides long-term glycemic control in type 2 diabetes. Diabetes Care. 2002 (Citado el 20 Abril del 2013). 2002; 47(2): 30-34. Disponible en: http://care. diabetesjournals.org/content/25/1/30.long

15. Burns N, Grove S. The practice of nursing research: Conduct, critique, and utilization. 5 ed. Philadelphia: Saunders; 2005.

16. Cohen S, Kamarck T, Mermelstein R. A global measure of perceived stress.Journal of Health and Social Behavior. 1983(Citado el 22 Abril del 2013); 24(4): 385-396. Disponible en: http://www.psy.cmu.edu/ scohen/ globalmeas83.pdf

17. Polonsky W, Fisher L, Mullan J, et al. Assesing psychosocial distress in diabetes. Diabetes Care. 2005 (Citado el 22
Abril del 2013); 28(3): 626-632. Disponible en: http://care. diabetesjournals.org/content/28/3/626.long

18. Glasgow R, Toober D, Guillete C. Psychosocial barriers to diabetes self-management and quality of life. Diabetes Espectrum. 2001(Citado el 20 Abril 2013); 14 (1): 33-14. Disponible en: http://spectrum.diabetesjournals. org/content/14/1/33.full.pdf+html

19. Alonso J, Regidor E, Barrio G, Prieto L, Rodríguez C, De La Fuente De Hoz L. Valores poblacionales de referencia de la versión española del Cuestionario de Salud SF-36. MedClin (Barc). 1998;111:410-416.

20. Clinical and Laboratory Standars Institute. Estandares para las necesidades de laboratorios clínicos y calidad en atención médica a nivel mundial. Wayne, PA: Clinical and LaboratoryStandardsInstitute; 2008. p. 168-186.

21. Secretaría de Salud, Instituto Nacional de Salud Pública. Norma Oficial Mexicana NOM-015-SSA2-2010 para la prevención, tratamiento y control de la diabetes mellitus. Mexico DF: Diario Oficial de la Federación;2010.

22. Ávila L, Cerón D, Ramos R, Velázquez L. Asociación del control glicémico con el apoyo familiar y el nivel de conocimientos en pacientes con diabetes tipo 2. Rev méd Chile. 2013 (Citado el 10 Agosto 2013); 141 (2): 173180. Disponible en: http://www.scielo.cl/scielo. php?pid=S0034-98872013000200005\&script=sci_arttext

23. Barquera S, Campos I, Hernández L, Pedroza A, Rivera J. Prevalencia de obesidad en adultos mexicanos, 20002012. Salud pública Méx. 2013 (Citado el 10 Agosto del 2013); 55 (S2): S151-S160. Disponible en: http://www. scielo.org.mx/scielo.php?pid=S003636342013000800012 \&script $=$ sci_arttext

24. Toledano C, Avila L, García S, Gómez H. Determinantes de adherencia terapéutica y control metabólico en pacientes ambulatorios con Diabetes mellitus tipo 2. Revista Mexicana de Ciencias Farmacéuticas. 2008 (Citado el 14 Agosto del 2013); 39(4): 9-17. Disponible en: http://www. redalyc.org/articulo.oa?id=57911113003

25. Dúran B, Rivera B, Franco E. Apego al tratamiento farmacológico en pacientes con diagnostico de diabetes mellitus tipo 2. Salud Pública Méx. 2008 (Citado el 14 Agosto del 2013); 43(3): 233-236. Disponible en: http:// bvs.insp.mx/rsp/articulos/articulo.php?id=001627

26. Orem D.Nursing: Concepts of practice. 6a ed. St. Louis: Mosby; 2001.

\section{Correspondencia:}

Milton Carlos Guevara Valtier

Facultad de Enfermería, Universidad Autónoma de Nuevo León

Av. José Eleuterio González (Gonzalitos 1500),

Mitras Centro, Monterrey, N.L.Mexico

Correo electrónico: carlos_valtier7@hotmail.com.mx

Fecha de Recepción: 15 de febrero del 2015

Fecha de aceptación: 15 de junio del 2015. 